\title{
Effect of Aronia melanocarpa (Black Chokeberry) supplementation on the development of obesity in mice fed a high-fat diet
}

\author{
Jamie I. Baum*, Luke R. Howard, Ronald L. Prior and Sun-Ok Lee \\ Department of Food Science, University of Arkansas, Fayetteville, AR, USA
}

Received 21 December 2015; accepted 29 March 2016

\begin{abstract}
.
BACKGROUND: Products derived from black chokeberry are claimed to be beneficial in treating chronic diseases, such as obesity and diabetes.

OBJECTIVE: The objective of this study was to determine if supplementation with Aronia melanocarpa (black chokeberry) juice concentrate (AJC) has anti-obesity properties in mice fed obesogenic diets.

METHODS: Male C57BL/6J mice ( $n=10$ /dietary treatment) were placed on either a low-fat, high-sucrose (LFHS; 5\% fat), LFHS+AJC (1.44 g AJC/kg diet), high-fat (HF; 30\% fat), or HF+AJC for 12-weeks.

RESULTS: Final body weight was lower in LFHS+AJC compared to LFHS, HF and HF+AJC $(\sim 14,20 \%$ and $\sim 16 \%$, respectively; $P<0.05)$. Mice receiving LFHS and LFHS+AJC had significantly higher $(P=0.001)$ energy intake than HF and HF+AJC. LFHS-fed mice had less $(-30 \%)$ epididymal fat $(p<0.05)$ than HF-fed mice, however mice on the LFHS+AJC had less epididymal fat per gram body weight than LFHS controls. There was no effect of diet or AJC on adipose tissue gene expression. There was no difference in plasma insulin, glucose or triglycerides between groups, however there was a positive effect of AJC on adiponectin $(P=0.059)$. There was also a significant effect of diet (LFHS versus HF) on HOMA-IR $(P=0.004)$ and HOMA-BCF $(P=0.002)$.

CONCLUSIONS: The results from this study demonstrate that AJC supplementation has the potential to prevent weight gain and markers of obesity. Further research is needed to determine mechanisms of action.
\end{abstract}

Keywords: Aronia melanocarpa, black chokeberry, anthocyanins, mice, obesity, diabetes, body fat

\section{Introduction}

The global prevalence of obesity has reached epidemic proportions in recent years [1]. Obese individuals have an increased risk of developing chronic diseases such as type 2 diabetes, hypertension and dyslipidemia [2]. Therefore, there is a need to develop successful nutritional interventions targeting obesity in order to treat or

\footnotetext{
*Corresponding author: Jamie I. Baum, PhD, Department of Food Science, University of Arkansas, 2650 North Young Avenue, Fayetteville, AR 72704, USA. Tel.: +1 479575 4474; Fax: +1 479575 6936; E-mail: baum@uark.edu. 
prevent weight gain and decrease risk for developing chronic disease. Epidemiological studies have demonstrated a correlation between consumption of fruits and vegetables with reduced incidence of obesity, diabetes and certain types of cancer [3-5], making increased consumption of fruits, such as berries, an ideal target for nutrition intervention. Berry fruits have shown considerable potential in the food industry and nutrition science community over the last two decades due to the high level of phenolic compounds (e.g. anthocyanins) they possess and the role these compounds play in maintaining health and preventing disease [6].

Aronia melanocarpa, also known as black chokeberry, is found in the eastern parts of North America and Northern and Eastern Europe. Products derived from black chokeberries are claimed to be beneficial in diseases associated with oxidative stress due to the high concentration of phenolic compounds (anthocyanins, procyanidins, hydroxycinnamic acids and flavonols) they possess [7-10]. Extracts from black chokeberries have been demonstrated to have anti-inflammatory [11], anticancer [12], cardio-protective [13] and anti-diabetic effects [14] in animal models. A recent study by Qin and Anderson [11] demonstrated that chokeberry extract prevented weight gain and decreased risk factors associated with insulin resistance in rats fed a fructose-rich diet through modulation of multiple pathways associated with insulin signaling, adipogenesis and inflammation [11]. In addition, supplementation with chokeberry juice reduced blood pressure and improved lipid status in both hypertensive individuals [15] and individuals with metabolic syndrome [16].

Although, there is emerging evidence regarding the ability of black chokeberries to prevent chronic disease, limited data exists on the potential for black chokeberry supplementation to prevent weight gain and the development of obesity. Therefore, the objective of this study was to determine if long-term supplementation with Aronia melanocarpa (black chokeberry) juice concentrate has anti-obesity properties in mice fed obesogenic diets.

\section{Materials and methods}

\subsection{Chemicals}

Aronia (black chokeberry) juice concentrate (AJC) ( $65^{\circ}$ brix) was obtained from Maes' Health and Wellness (Omaha, NE).

\subsection{HPLC analysis of anthocyanins, flavonols and hydroxycinnamic acids}

Following dilution of Aronia juice concentrate $\left(65^{\circ}\right.$ brix) to single strength juice $\left(12^{\circ}\right.$ brix) with MilliQ water, $8 \mathrm{~mL}$ of juice was evaporated to dryness using a SpeedVac ${ }^{\circledR}$ concentrator (ThermoSavant, Holbrook, NY) and re-suspended in $1 \mathrm{~mL}$ of $3 \%$ formic acid in water. Individual anthocyanins, flavonols and chlorogenic acid were separated by reverse phase HPLC on a Symmetry $\mathrm{C}_{18}$ column (Water Corp., Milford, MA) according to the method described by Cho et al. (2004). Anthocyanins, flavonols and hydroxycinnamic acids were identified by comparing retention times of peaks that were previously identified by HPLC/MS using identical HPLC methodology [17, 18]. Anthocyanin glycosides were quantified as corresponding anthocyanin glucosides using external calibration curves of a mixture of anthocyanin glucoside standards purchased from Polyphenols Laboratories (Sandnes, Norway). Flavonol glycosides were quantified as rutin equivalents and hydroxycinnamic acids were quantified as chlorogenic acid equivalents.

\subsection{HPLC analysis of procyanidins}

Samples (20 mL) of Aronia juice concentrate diluted to single strength juice with Milli-Q water were subjected to solid-phase extraction using Sephadex LH-20 to remove interfering sugars, and other phenolic compounds according to the method described by Gu et al. [19]. Procyanidin extract eluted from the column with $80 \mathrm{~mL}$ of 
$70: 30$ acetone:water (v/v) was evaporated to dryness using a SpeedVac ${ }^{\circledR}$ concentrator, re-suspended in $2 \mathrm{~mL}$ of acetone/water/acetic acid $(70: 29.5: 0.5 \mathrm{v} / \mathrm{v} / \mathrm{v})$, and filtered through $0.45 \mu \mathrm{m}$ filters. Procyanidins were separated by normal phase HPLC on a Phenomenex Luna column (Torrance, CA) according to the method of Hammerstone et al. [20]. Peaks were quantified using external calibration curves of a mixture of procyanidin standards (DP1 - DP6) isolated from cocoa and provided by Masterfoods (Hackettstown, NJ).

\subsection{Animals and diets}

Forty, 6-week old, male C57BL/6J mice (Jackson Laboratories, Bar Harbor, MN) were used in this study. Two obesogenic diets were designed and used for this study: Low-Fat, High-Sucrose (LFHS) and High-Fat (HF; $55 \%$ energy from fat) supplemented with or without AJC. Mice were fed one of four dietary treatments (Table 1) LFHS, LFHS+AJC, HF, or HF+AJC, for 12 weeks. The AJC was added as liquid concentrate (60\% solids) to the powdered diet ( $1.44 \mathrm{~g}$ anthocyanins per $\mathrm{kg}$ diet or the equivalent of 97 Aronia berries ( $~ 0.5 \mathrm{cups})$ ). Animals were randomly assigned to a diet treatment group before the start of the study. Animals were housed two per cage with a 12-hour light/dark cycle with ad libitum access to food and water. Body weights and food intake were measured three times per week, however food spillage was not measured. At the end of the 12-week experimental period, mice were fasted overnight ( $8-10$ hours) and euthanized by cervical dislocation followed by cardiac puncture to collect blood. The experimental protocol was approved by the Institutional Animal Care and Use Committee at the University of Arkansas (Fayetteville, AR).

Diet efficiency was calculated by dividing average daily weight gain by average daily energy intake in kilocalories (kcal). Blood was collected in EDTA-lined tubes (BD Vacutainer) and centrifuged at $1,800 \times g$ for $10 \mathrm{~min}$ at $4{ }^{\circ} \mathrm{C}$. Plasma was collected and immediately placed in liquid nitrogen and stored at $-80^{\circ} \mathrm{C}$ until analysis. Epididymal fat pads and liver samples were collected and weighed and immediately frozen in liquid nitrogen and stored at $-80^{\circ} \mathrm{C}$. The cecum was removed after sacrifice, weighed and the cecum contents were removed and weighed separately. The cecum contents were immediately frozen and stored at $-80^{\circ} \mathrm{C}$.

\subsection{Plasma analysis}

All plasma analyses were conducted using fasting plasma samples and commercially available kits (Cayman Chemical, Ann Arbor, MI). Fasting plasma glucose (item \#10009582) and triglyceride levels (item \#10010303) were measured using a commercially available colorimetric assay kit. Fasting insulin (item \#589501), and

Table 1

Experimental diet composition

\begin{tabular}{lcccc}
\hline & LFHS & LFHS+AJC & HF & HF+AJC \\
\hline Casein, g & 204 & 204 & 204 & 204 \\
Mineral Mix*, g & 35 & 35 & 10 & 35 \\
Vitamin Mix*, g & 10 & 10 & 2 & 10 \\
Choline Chloride, g & 2 & 2 & 50 & 2 \\
Corn Oil, g & 50 & 50 & 300 & 50 \\
Lard, g & 0 & 0 & 40 & 300 \\
Cellulose Powder, & 40 & 40 & 0 & 40 \\
Aronia juice & 0 & 193 & 309 & 354.18 \\
concentrate, g & 659 & 654.18 & & \\
Sucrose, g & & & & \\
\hline
\end{tabular}

*Based on AIN-93M diet vitamin and mineral content. LFHS: Low-fat, high-sucrose; AJC: Aronia juice concentrate; HF: High-fat. 
adiponectin (item \#10007620) levels were measured using commercially available enzyme immunoassays. The homeostasis model assessment (HOMA) for insulin resistance (HOMA-IR) and for $\beta$-cell function (HOMA$\mathrm{BCF})$ were calculated as described previously [21]: HOMA-IR $=($ fasting glucose $(\mathrm{mg} / \mathrm{dL}) \times$ fasting insulin $(\mu \mathrm{IU} / \mathrm{ml})) / 405$ and HOMA-BCF $=(360 \times$ fasting insulin $) /($ fasting glucose -63$)$.

\subsection{Gene expression}

RNA was isolated from adipose tissue according to manufacturer's instructions using Trizol (Life Technologies, Grand Island, NY). Quanta Biosciences (Gaithersburg, MD) qScript cDNA synthesis kit was used to make cDNA according to manufacturer's instructions. All genes were analyzed by qRT-PCR using a LightCycler 480 (Roche Life Science, Indianapolis, IN) using Roche RealTime Ready custom assays for fatty acid synthase (FAS) and peroxisome-proliferator receptor gamma (PPAR $\gamma$ ). All data was normalized to the housekeeping gene GAPDH.

\subsection{Statistical analysis}

All data were analyzed using Prism GraphPad Software (La Jolla, CA). $P$ values $<0.05$ were considered significant. One-way ANOVA followed by a Tukey test to correct for multiple comparisons was used for analyzing differences between means. Two-way ANOVA followed by a Bonferroni test to correct for multiple comparisons was used to analyze diet-AJC interactions, and effects of diet and AJC supplementation.

\section{Results}

\subsection{Polyphenol composition of Aronia (black chokeberry) juice concentrate}

The concentrations of individual anthocyanins and procyanidins and total flavonols and hydroxycinnamic acids in Aronia juice concentrate (AJC) are shown in Table 2. The composition of anthocyanins (expressed as single strength juice (957.2 (Table 2)/5.4)) is similar to previous reports [7, 17]. The content of total anthocyanins $(177 \mathrm{mg} / \mathrm{mL})$ in AJ was similar to the value of $169 \mathrm{mg} / 100 \mathrm{~mL}$ previously reported for AJ prepared from AJC [17].

\subsection{Body weight, food intake and tissue weight}

There was no difference in starting body weights between groups (Table 3). There was a significant effect $(P<0.05)$ dietary fat composition and AJC supplementation $(P<0.01)$ on final body weight. At the end of the 12-week dietary intervention, animals in the HF and HF+AJC groups had significantly higher $(P<0.05)$ body weights than animals in the LFHS+AJC group. There was a trend $(P=0.055)$ for the LFHS+AJC group to have lower body weights than the LFHS group. AJC supplementation had a significant effect $(P<0.001)$ on weight gain and animals supplemented with AJC tended to gain less weight. Animals consuming the LFHS and LFHS+AJC diets had significantly higher $(P<0.001)$ energy intake $(\mathrm{kcal} / \mathrm{d})$ compared to the HF and $\mathrm{HF}+\mathrm{AJC}$ groups, however this was due to the fat content of the diet and not the AJC. There was a significant $(P<0.05)$ diet-AJC supplementation interaction on diet efficiency and both diet $(P<0.0001)$ and AJC supplementation $(P=0.02)$ had a significant effect on diet efficiency, demonstrating the AJC supplementation reduced diet efficiency. A lower diet efficiency is desirable since it demonstrates that the animals need less energy intake to maintain body processes (i.e. growth) and are more efficient at using the energy they consume.

Liver weight per gram body weight was significantly lower $(P<0.05)$ in $\mathrm{HF}$ and $\mathrm{HF}+\mathrm{AJC}$ groups compared to the LFHS and LFHS+AJC groups (Fig. 1A), which could be due to the high sucrose content of the LFHS diets. In addition, epididymal fat weight per gram body weight was significantly lower in LFHS and LFHS+AJC groups 
Table 2

Polyphenol composition of Aronia juice concentrate (AJC)

\begin{tabular}{lc}
\hline Compound $(\mathrm{mg} / 100 \mathrm{~g})$ & AJC \\
\hline Total monomeric anthocyanins & 957.2 \\
cyanidin-3-glactoside & 733.3 \\
cyanidin-3-glucoside & 34.1 \\
cyanidin-3-arabinoside & 187.9 \\
pelargonidin-3-arabinoside & 7.6 \\
Total procyanidins & 532.8 \\
monomers & 60.0 \\
dimers & 101.5 \\
trimers & 69.6 \\
tetramers & 83.4 \\
pentamers & 68.8 \\
hexamers & 129.9 \\
heptamers & 60.7 \\
Total flavonols & 308.9 \\
Total hydroxycinnamic acids & 271 \\
\hline
\end{tabular}

Table 3

Body weight and food intake

\begin{tabular}{|c|c|c|c|c|c|c|c|}
\hline & \multirow[b]{2}{*}{ LFHS } & \multirow[b]{2}{*}{ LFHS+AJC } & \multirow[b]{2}{*}{$\mathrm{HF}$} & \multirow[b]{2}{*}{$\mathrm{HF}+\mathrm{AJC}$} & \multicolumn{3}{|c|}{$P$ value } \\
\hline & & & & & $\begin{array}{c}\text { Diet-AJC } \\
\text { Interaction }\end{array}$ & $\begin{array}{c}\text { Effect of } \\
\text { Diet }\end{array}$ & $\begin{array}{c}\text { Effect of } \\
\text { AJC }\end{array}$ \\
\hline \multicolumn{8}{|l|}{ Body Weight (g) } \\
\hline Intial & $15.77 \pm 0.55^{\mathrm{a}}$ & $14.01 \pm 0.50^{\mathrm{a}}$ & $13.90 \pm 0.60^{\mathrm{a}}$ & $14.34 \pm 0.58^{\mathrm{a}}$ & & & \\
\hline Final & $35.53 \pm 0.91^{\mathrm{ab}}$ & $31.29 \pm 1.09^{\mathrm{a}}$ & $37.76 \pm 1.49^{\mathrm{b}}$ & $36.28 \pm 1.21^{\mathrm{b}}$ & 0.298 & 0.035 & 0.009 \\
\hline Total Weight Gain (g) & $19.76 \pm 0.91^{\mathrm{ab}}$ & $17.28 \pm 0.86^{\mathrm{a}}$ & $23.86 \pm 1.46^{\mathrm{b}}$ & $21.94 \pm 1.10^{\mathrm{b}}$ & 0.801 & 0.055 & $<0.001$ \\
\hline \multicolumn{8}{|l|}{ Daily Food Intake } \\
\hline $\mathrm{g} / \mathrm{d}$ & $5.45 \pm 0.50^{\mathrm{a}}$ & $4.48 \pm 0.49^{\mathrm{a}}$ & $2.50 \pm 0.15^{\mathrm{b}}$ & $2.70 \pm 0.19^{b}$ & 0.099 & $<0.0001$ & 0.291 \\
\hline $\mathrm{kcal} / \mathrm{d}$ & $20.92 \pm 2.11^{\mathrm{a}}$ & $18.57 \pm 2.00^{\mathrm{ab}}$ & $13.53 \pm 0.77^{\mathrm{b}}$ & $15.28 \pm 1.03^{\mathrm{ab}}$ & 0.134 & 0.001 & 0.742 \\
\hline Diet Efficiency & $1.69 \pm 0.04^{\mathrm{a}}$ & $1.68 \pm 0.06^{\mathrm{a}}$ & $2.79 \pm 0.11^{\mathrm{b}}$ & $1.37 \pm 0.08^{\mathrm{c}}$ & 0.022 & $<0.0001$ & 0.015 \\
\hline
\end{tabular}

Data expressed as means \pm SEM. Means not sharing the same letter are significantly different (one-way ANOVA; $P<0.05$ ). Diet-AJC interaction determined by two-way ANOVA $(P<0.05)$. LFHS: Low-fat, high-sucrose; AJC: Aronia juice concentrate; HF: High-fat.

$(P<0.05)$ compared to the HF and HF+AJC groups (Fig. 1B). There was a trend $(P=0.06)$ for LFHS+AJC to have lower epididymal fat weight compared to LFHS. Finally, the cecum contents weighed significantly more $(P<0.05)$ in the $\mathrm{LF}+\mathrm{AJC}$ and the HF+AJC groups compared to the LF and HF groups (Fig. 1C).

\subsection{Metabolic markers of obesity}

Metabolic plasma markers of obesity are shown in Table 4. There was no difference between groups for fasting glucose, insulin and triglyceride levels. However, there was a trend $(P=0.059)$ for AJC supplementation 

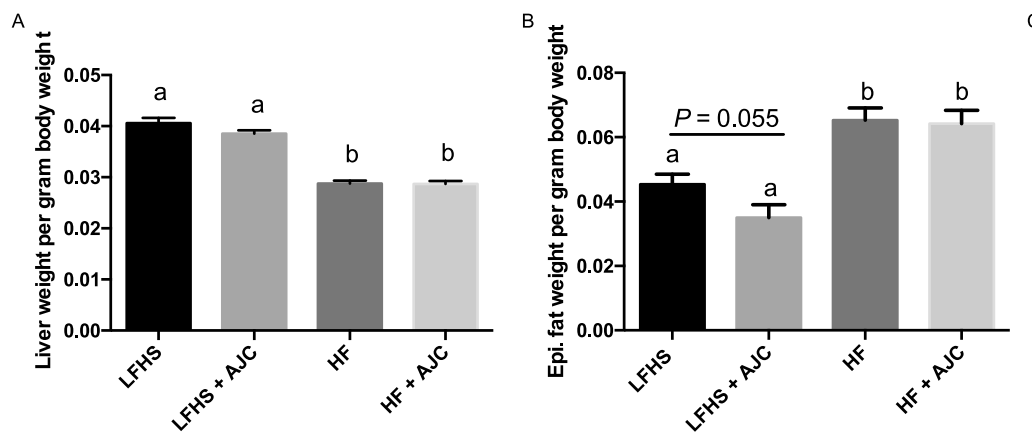

C

Fig. 1. The effect of Aronia juice supplementation on liver weight, epididymal fat, and cecum contents. A. Liver weight per gram body weight. $B$. Epididymal fat pad weight per gram body weight. $C$. Weight of cecum contents. Data expressed as means + SEM. Means not sharing the same letter are significantly different (one-way ANOVA; $P<0.05$ ). LFHS: Low-fat, high-sucrose; AJC: Aronia juice concentrate; HF: High-fat.

Table 4

Metabolic markers of obesity

\begin{tabular}{lccccccc}
\hline & & & & & \multicolumn{2}{c}{$P$ value } \\
\cline { 3 - 7 } & LFHS & LFHS+AJC & HF & HF+AJC & $\begin{array}{c}\text { Diet-AJC } \\
\text { Interaction }\end{array}$ & $\begin{array}{c}\text { Effect of } \\
\text { Diet }\end{array}$ & $\begin{array}{c}\text { Effect of } \\
\text { AJC }\end{array}$ \\
\hline Glucose (mg/dL) & $126.7 \pm 15.1$ & $207.4 \pm 17.7$ & $169.1 \pm 15.0$ & $161.8 \pm 10.2$ & 0.23 & 0.066 & 0.472 \\
Insulin (pmol/L) & $80.2 \pm 11.0$ & $67.6 \pm 12.1$ & $111.4 \pm 23.2$ & $109.9 \pm 34.1$ & 0.825 & 0.156 & 0.778 \\
Triglycerides (mg/dL) & $101.0 \pm 14.3$ & $96.7 \pm 9.9$ & $89.6 \pm 5.0$ & $103.0 \pm 5.7$ & 0.359 & 0.787 & 0.638 \\
Adiponectin (ng/ml) & $6.6 \pm 2.3$ & $10.6 \pm 2.3$ & $6.9 \pm 2.2$ & $11.9 \pm 2.2$ & 0.819 & 0.727 & 0.059 \\
HOMA-IR & $25.4 \pm 3.0$ & $34.6 \pm 2.9$ & $46.5 \pm 4.1$ & $43.9 \pm 2.8$ & 0.787 & 0.004 & 0.609 \\
HOMA-BCF & $214.8 \pm 87.7^{\text {ab }}$ & $224.0 \pm 64.5^{\text {a }}$ & $475.6 \pm 67.6^{\mathrm{b}}$ & $449.3 \pm 5605^{\mathrm{b}}$ & 0.743 & 0.002 & 0.445 \\
\hline
\end{tabular}

Data expressed as means \pm SEM. Diet-AJC interaction determined by two-way ANOVA $(P<0.05)$. LF: Low-fat, high-sucrose; AJC: Aronia juice concentrate; HF: High-fat.

Table 5

Adipose tissue gene expression

\begin{tabular}{lcccr}
\hline & LFHS & LFHS+AJC & HF & HF+AJC \\
\hline FAS & $1.19 \pm 0.31$ & $1.11 \pm 0.21$ & $1.05 \pm 0.15$ & $1.01 \pm 0.06$ \\
PPAR $\gamma$ & $1.17 \pm 0.32$ & $1.44 \pm 0.39$ & $1.03 \pm 0.12$ & $1.48 \pm 0.37$ \\
\hline
\end{tabular}

Gene expression data is expressed as mean $2^{\Delta \Delta C T} \pm$ SEM. LF: Low-fat, high-sucrose; AJC: Aronia juice concentrate; HF: High-fat; FAS: fatty acid synthase; $\operatorname{PPAR}_{\gamma}$ : peroxisome proliferator-activated receptor gamma.

to increase adiponectin levels. There was no difference in HOMA-IR and HOMA-BCF between groups, however there was a significant effect of diet on both HOMA-IR $(P=0.004)$ and HOMA-BCF $(P=0.002)$. There was no difference in adipose tissue gene expression of FAS and PPAR $\gamma$ between groups (Table 5). 


\section{Discussion}

This is the first study to compare the effect of AJC supplementation on body weight, body composition and metabolic markers of obesity. The present study suggests that AJC supplementation has the potential to prevent weight gain and markers of obesity in a rodent model by decreasing weight gain and increasing levels of adiponectin. In addition, animals in this study were fed two different obesogenic diets, which manifested themselves in different ways. Animals fed the LFHS had higher liver weights and lower total weight gain, while the HF-fed mice had higher weight gain and higher levels of epididymal fat.

There was a trend for higher plasma levels of adiponectin with AJC supplementation. Adiponectin is the most abundant peptide secreted by adipocytes and is a key hormone in the inter-relationship between adiposity, insulin resistance and inflammation [22]. Circulating adiponectin levels are inversely related to body fat percentage [23]. Adiponectin increases the sensitivity to insulin through activating AMP kinase, promoting glucose uptake by muscles via intracellular translocation of the GLUT4 transporters and by reducing gluconeogenesis by inhibiting the hepatic enzyme phosphoenolpyruvate carboxylase, which inhibits the synthesis of fatty acids and stimulates their oxidation [22]. Our data support results from an earlier study, which demonstrates that chokeberry extract elevated plasma adiponectin in rats fed a high-fructose diet when compared with the control group [11].

Peroxisome-proliferator receptor gamma (PPAR $\gamma$ ) is a gene that regulates fatty acid and lipid metabolism [24]. In this study, we found no change in PPAR $\gamma$ expression in epididymal fat tissue with AJC supplementation. However, a recent study by Qin and Anderson [11] showed that animals fed a high-fructose diet supplemented with chokeberry extract in the drinking water for five weeks had increased PPAR $\gamma$ expression in epididymal fat.

In the present study, dietary supplementation with AJC did not appear to alter insulin resistance since AJC did not have any significant effect on HOMA-IR, HOMA-BCF or fasting insulin and glucose levels. However, HOMA-IR was increased in mice fed the HF diets. In studies by Heyman and Axling [25], mice fed a high fat diet (45 kcal\% fat) and either lingonberries, blackcurrants, or bilberries, had a lower HOMA-IR index resulting from a lower fasting glucose and/or insulin, indicating that these three berries protected against high-fat induced insulin resistance. The diet supplemented with lingonberries was more effective than the other berries in that it provided an almost complete prevention of body weight gain which was an effect of reduced adiposity [25]. Body weight and epididymal fat gain was not changed with AJC supplementation in this study indicating that chokeberries may not exhibit the same effect as the berries described above.

In this study, there was a trend for AJC supplementation to reduce weight gain and adiposity in mice fed a LFHS diet, with no effect in mice fed a high-fat diet. The reasons for the apparent lack of consistency of results found in various studies related to the effectiveness of berries on obesity and obesity-related parameters are not clear. The type of diet used to induce obesity, the amount of berry in the diet, the format of supplementation (e.g. extract versus whole berry), and polyphenolic composition of the different berries are all possible factors. In the study by Heyman and Axling [25], 20\% of the diet in the berry experimental groups came from freeze dried berries. Earlier studies comparing whole versus powdered blueberries or strawberries found that the whole berries were not effective in preventing obesity $[26,27]$ and, in some cases, tended to promote obesity in the context of a high-fat diet but not with a low-fat diet. Other studies have also reported either increased obesity [28] or equivalent adiposity [29] with supplementing a high-fat diet with whole blueberry powder at 2 or $4 \%$ of the total diet weight. Whole, freeze-dried black raspberry powder was also ineffective in preventing obesity [30]. In addition, whole strawberries did not promote obesity when supplemented in a high-fat diet, and some measures indicated possible antiobesity effects [26, 30-34]. However, feeding purified anthocyanins in the drinking water from blueberries $(2.8 \mathrm{mg} /$ day) or strawberries has been shown to reduce obesity in a rodent model [31]. One issue could be the anthocyanin concentration in the diets. However, the anthocyanin concentration in this study $(1.44 \mathrm{~g} / \mathrm{kg}$ diet $)$ was comparable to published literature on purple corn $(2 \mathrm{~g} / \mathrm{kg}$ diet $)$, blueberry powder $(2.43 \mathrm{~g} / \mathrm{kg}$ diet) [27], and cornelian cherry ( $1 \mathrm{~g} / \mathrm{kg}$ diet) [35], and higher than studies using blueberry powder at lower concentrations ( $260 \mathrm{mg} / \mathrm{kg}$ diet) [26] and strawberry powder (33 mg/kg diet) [36]. The mechanisms as to why 
supplementation of purified anthocyanins delays the development of obesity and supplementation with the whole berry does not prevent obesity still need to be elucidated.

There was no effect of AJC supplementation on plasma triglyceride levels. This is in contrast to previous studies from our lab, which found that purified berry (blueberry and strawberry) anthocyanins supplemented in the drinking water, but not whole berries, decreased serum cholesterol and triglyceride level [26]. In addition, hypertensive adults who were fed chokeberry juice daily for four weeks showed significant reductions in triglyceride levels, total and LDL cholesterol and systolic and diastolic blood pressure [15]. Similar effects were observed in patients with metabolic syndrome who were supplemented with chokeberry juice for two months [16]. These differences could be due to bioavailability and method of administration of the supplement [37-39]. Furthermore, epidemiological data suggests that a high intake of anthocynins may reduce risk of myocardial infarctions, suggesting improved heart health [32]. In our study, AJC was incorporated into the powdered diet whereas the rodent study administered the purified berries in the drinking water and human studies the subjects were administered the berries in juice format. In addition, the studies discussed above were conducted in health-compromised individuals, while the mice were not health-compromised in this study.

Finally, the weight of the content in the cecum was higher with AJC supplementation. However, in a study by Frejnagel and Juskiewicz [40] differences in weights of the cecal contents were not observed with a chokeberry extract, but when an extract of polyphenols from green tea was fed, an increase in cecal content weight was observed [40]. In another study, feeding the polyphenol extract from chokeberries altered cecal parameters and metabolism as shown by a reduction in cecal digesta $\mathrm{pH}$ and ammonia concentrations in comparison to control animals [40]. In a previous study by Heyman and Axling [25] in which diets were supplemented with one of 8 different berries the mass of the cecum, including its contents, was increased in all berry supplemented groups compared to the control diet with the exception of the raspberry supplemented group [25]. Our data support these findings, which could be due a change in microbial fermentative activity in the cecum [41], however further research is needed to determine the mechanisms of action.

This study has several limitations. The high fat diet used in this study contained only $30 \%$ fat, while previously published studies have used $45 \%$ to $60 \%$ fat $[31,42]$. Using a lower level of fat in the high-fat diet in this study may have influenced the amount of weight the animals gained and made metabolic parameters related to obesity less pronounced compared to studies using higher levels of fat in the diet. Additionally, some studies have studied the effect of berry supplementation on animals fed a high-fructose diet [11], which may have differing effects on metabolism when compared to high-fat feeding. In addition, one of our obesogenic diets, LFHS, was high in sucrose. High sucrose diets have been used to induce obesity and diabetes in rodent models [43-46]. The high levels of sucrose in the LFHS diets could explain why we did not find large differences between LFHS and HF groups. Additionally, the diets vary in percent of energy contributed by protein $(21 \%$ in the LFHS and $16.4 \%$ in the HF), which could have impacted our results. Finally, we supplemented the AJC directly into the powdered diet, whereas the other chokeberry supplementation studies added the chokeberry concentrate to the drinking water. These differences in dietary manipulation may be responsible for the varying results.

In summary, this study demonstrates that supplementation with AJC increased adiponectin levels and reduced weight gain and body fat in mice fed a low-fat diet. However, there was no difference in fasting glucose, insulin or triglyceride levels with AJC supplementation. Further research is needed to define the health benefits of AJC supplementation and understand the underlying mechanisms of action.

\section{Acknowledgments}

This study was supported by a grant from the Arkansas Biosciences Institute. 


\section{References}

[1] Ogden CL, Carroll MD, Kit BK, Flegal KM. Prevalence of childhood and adult obesity in the United States, 2011-2012. JAMA. 2014;311:806-14.

[2] Cope MB, Allison DB. Obesity: Person and population. Obesity (Silver Spring). 2006;14(Suppl 4):156S-9S.

[3] Holtan SG, O'Connor HM, Fredericksen ZS, Liebow M, Thompson CA, Macon WR, et al. Food-frequency questionnaire-based estimates of total antioxidant capacity and risk of non-Hodgkin lymphoma. Int J Cancer. 2012;131:1158-68.

[4] Murthy NS, Mukherjee S, Ray G, Ray A. Dietary factors and cancer chemoprevention: An overview of obesity-related malignancies. J Postgrad Med. 2009;55:45-54.

[5] Rissanen TH, Voutilainen S, Virtanen JK, Venho B, Vanharanta M, Mursu J, et al. Low intake of fruits, berries and vegetables is associated with excess mortality in men: The Kuopio Ischaemic Heart Disease Risk Factor (KIHD) Study. J Nutr. 2003;133:199-204.

[6] Meydani M, Hasan ST. Dietary polyphenols and obesity. Nutrients. 2010;2:737-51.

[7] Wu X, Gu L, Prior RL, McKay S. Characterization of anthocyanins and proanthocyanidins in some cultivars of Ribes, Aronia, and Sambucus and their antioxidant capacity. J Agric Food Chem. 2004;52:7846-56.

[8] He J, Giusti MM. Anthocyanins: Natural colorants with health-promoting properties. Annu Rev Food Sci Technol. 2010;1:163-87.

[9] Giampieri F, Alvarez-Suarez JM, Battino M. Strawberry and human health: Effects beyond antioxidant activity. J Agric Food Chem. 2014;62:3867-76.

[10] Pistollato F, Giampieri F, Battino M. The use of plant-derived bioactive compounds to target cancer stem cells and modulate tumor microenvironment. Food Chem Toxicol. 2015;75:58-70.

[11] Qin B, Anderson RA. An extract of chokeberry attenuates weight gain and modulates insulin, adipogenic and inflammatory signalling pathways in epididymal adipose tissue of rats fed a fructose-rich diet. Br J Nutr. 2012;108:581-7.

[12] Szaefer H, Krajka-Kuzniak V, Ignatowicz E, Adamska T, Baer-Dubowska W. Chokeberry (Aronia melanocarpa) juice modulates 7,12-dimethylbenz[a]anthracene induced hepatic but not mammary gland phase I and II enzymes in female rats. Environ Toxicol Pharmacol. 2011;31:339-46.

[13] Valcheva-Kuzmanova S, Kuzmanov K, Mihova V, Krasnaliev I, Borisova P, Belcheva A. Antihyperlipidemic effect of Aronia melanocarpa fruit juice in rats fed a high-cholesterol diet. Plant Foods Hum Nutr. 2007;62:19-24.

[14] Jurgonski A, Juskiewicz J, Zdunczyk Z. Ingestion of black chokeberry fruit extract leads to intestinal and systemic changes in a rat model of prediabetes and hyperlipidemia. Plant Foods Hum Nutr. 2008;63:176-82.

[15] Kardum N, Milovanovic B, Savikin K, Zdunic G, Mutavdzin S, Gligorijevic T, et al. Beneficial effects of polyphenol-rich chokeberry juice consumption on blood pressure level and lipid status in hypertensive subjects. J Med Food. 2015;18:1231-8.

[16] Broncel M, Kozirog M, Duchnowicz P, Koter-Michalak M, Sikora J, Chojnowska-Jezierska J. Aronia melanocarpa extract reduces blood pressure, serum endothelin, lipid, and oxidative stress marker levels in patients with metabolic syndrome. Med Sci Monit. 2010;16:CR28-34.

[17] Howard LR, Brownmiller C, Prior RL, Mauromoustakos A. Improved stability of chokeberry juice anthocyanins by beta-cyclodextrin addition and refrigeration. J Agric Food Chem. 2013;61:693-9.

[18] Cho MJ, Howard LR, Prior RL, Clark JR. Flavonol glycosides and antioxidant capacity of various blackberry and blueberry genotypes determined by high-performance liquid chromatography/mass spectrometry. J Sci Food Agric. 2005;85:2149-58.

[19] Gu L, Kelm MA, Hammerstone JF, Zhang Z, Beecher G, Holden J, et al. Liquid chromatographic/electrospray ionization mass spectrometric studies of proanthocyanidins in foods. J Mass Spectrom. 2003;38:1272-80.

[20] Hammerstone JF, Lazarus SA, Mitchell AE, Rucker R, Schmitz HH. Identification of procyanidins in cocoa (Theobroma cacao) and chocolate using high-performance liquid chromatography mass spectrometry. J Agric Food Chem. 1999;47:490-6.

[21] Prior RL, E Wilkes S, Rogers T, Khanal RC, Wu X, Howard LR. Purified blueberry anthocyanins and blueberry juice alter development of obesity in mice fed an obesogenic high-fat diet. J Agric Food Chem. 2010;58:3970-6.

[22] Fisman EZ, Tenenbaum A. Adiponectin: A manifold therapeutic target for metabolic syndrome, diabetes, and coronary disease? Cardiovascular diabetology. 2014;13:103.

[23] Ukkola O, Santaniemi M. Adiponectin: A link between excess adiposity and associated comorbidities? J Mol Med (Berl). 2002;80:696702.

[24] Jones JR, Barrick C, Kim KA, Lindner J, Blondeau B, Fujimoto Y, et al. Deletion of PPARgamma in adipose tissues of mice protects against high fat diet-induced obesity and insulin resistance. PNAS. 2005;102:6207-12.

[25] Heyman L, Axling U, Blanco N, Sterner O, Holm C, Berger K. Evaluation of beneficial metabolic effects of berries in high-fat fed C57BL/6J mice. J Nutr Metab. 2014;2014:403041.

[26] Prior RL, Wu X, Gu L, Hager T, Hager A, Wilkes S, et al. Purified berry anthocyanins but not whole berries normalize lipid parameters in mice fed an obesogenic high fat diet. Mol Nutr Food Res. 2009;53:1406-18. 
[27] Prior RL, Wu X, Gu L, Hager TJ, Hager A, Howard LR. Whole berries versus berry anthocyanins: Interactions with dietary fat levels in the C57BL/6J mouse model of obesity. J Agric Food Chem. 2008;56:647-53.

[28] Wu D, Ren Z, DeFuria J, Obin M, Meydani SN. Effect of blueberries on the immune response of obese mice induced by high fat diet. FASEB J. 2009;23:908.5.

[29] DeFuria J, Bennett G, Strissel KJ, Perfield JW 2nd, Milbury PE, Greenberg AS, et al. Dietary blueberry attenuates whole-body insulin resistance in high fat-fed mice by reducing adipocyte death and its inflammatory sequelae. J Nutr. 2009;139:1510-6.

[30] Prior RL, Wu X, Gu L, Hager T, Hager A, Wilkes S, et al. Purified berry anthocyanins but not whole berries normalize lipid parameters in mice fed an obesogenic high fat diet. Mol Nutr Food Res. 2009;53:1406-18.

[31] Prior RL, Wu X, Gu L, Hager TJ, Hager A, Howard LR. Whole berries versus berry anthocyanins: Interactions with dietary fat levels in the C57BL/6J mouse model of obesity. J Agric Food Chem. 2008;56:647-53.

[32] Cassidy A, Mukamal KJ, Liu L, Franz M, Eliassen AH, Rimm EB. High anthocyanin intake is associated with a reduced risk of myocardial infarction in young and middle-aged women. Circulation. 2013;127:188-96.

[33] Alvarez-Suarez JM, Giampieri F, Tulipani S, Casoli T, Di Stefano G, Gonzalez-Paramas AM, et al. One-month strawberry-rich anthocyanin supplementation ameliorates cardiovascular risk, oxidative stress markers and platelet activation in humans. J Nutr Biochem. 2014;25:289-94.

[34] Basu A, Fu DX, Wilkinson M, Simmons B, Wu M, Betts NM, et al. Strawberries decrease atherosclerotic markers in subjects with metabolic syndrome. Nutr Res. 2010;30:462-9.

[35] Jayaprakasam B, Olson LK, Schutzki RE, Tai MH, Nair MG. Amelioration of obesity and glucose intolerance in high-fat-fed C57BL/6 mice by anthocyanins and ursolic acid in Cornelian cherry (Cornus mas). J Agric Food Chem. 2006;54:243-8.

[36] Tsuda T, Horio F, Uchida K, Aoki H, Osawa T. Dietary cyanidin 3-O-beta-D-glucoside-rich purple corn color prevents obesity and ameliorates hyperglycemia in mice. J Nutr. 2003;133:2125-30.

[37] Charron CS, Kurilich AC, Clevidence BA, Simon PW, Harrison DJ, Britz SJ, et al. Bioavailability of anthocyanins from purple carrot juice: Effects of acylation and plant matrix. J Agric Food Chem. 2009;57:1226-30.

[38] McGhie TK, Walton MC. The bioavailability and absorption of anthocyanins: Towards a better understanding. Mol Nutr Food Res. 2007;51:702-13.

[39] Yang M, Koo SI, Song WO, Chun OK. Food matrix affecting anthocyanin bioavailability: Review. Curr Med Chem. 2011;18:291-300.

[40] Frejnagel S, Juskiewicz J. Dose-dependent effects of polyphenolic extracts from green tea, blue-berried honeysuckle, and chokeberry on rat caecal fermentation processes. Planta Med. 2011;77:888-93.

[41] Kishino E, Ito T, Fujita K, Kiuchi Y. A mixture of Salacia reticulata (Kotala himbutu) aqueous extract and cyclodextrin reduces body weight gain, visceral fat accumulation, and total cholesterol and insulin increases in male Wistar fatty rats. Nutr Res. 2009;29:55-63.

[42] Tsuda T, Horio F, Uchida K, Aoki H, Osawa T. Dietary cyanidin 3-O-beta-D-glucoside-rich purple corn color prevents obesity and ameliorates hyperglycemia in mice. J Nutr. 2003;133:2125-30.

[43] Basaranoglu M, Kayacetin S, Yilmaz N, Kayacetin E, Tarcin O, Sonsuz A. Understanding mechanisms of the pathogenesis of nonalcoholic fatty liver disease. World J Gastroenterol. 2010;16:2223-6.

[44] Shafrir E, Ziv E, Kalman R. Nutritionally induced diabetes in desert rodents as models of type 2 diabetes: Acomys cahirinus (spiny mice) and Psammomys obesus (desert gerbil). ILAR J. 2006;47:212-24.

[45] Harzallah A, Hammami M, Kepczynska MA, Hislop DC, Arch JR, Cawthorne MA, et al. Comparison of potential preventive effects of pomegranate flower, peel and seed oil on insulin resistance and inflammation in high fat and high sucrose diet-induced-obesity mice model. Arch Physiol Biochem. 2016:1-40.

[46] Kouno T, Akiyama N, Ito T, Okuda T, Nanchi I, Notoya M, et al. Ghrelin O-acyltransferase knockout mice show resistance to obesity when fed high-sucrose diet. J Endocrinol. 2016;228:115-25. 